\title{
Endoscopic ultrasound-guided ethanol ablation of a large metastatic carcinoid tumor: success with a note of caution
}

Authors

Institutions
Bradley W. Mathers ${ }^{1}$, Harold A. Harvey ${ }^{2}$, Charles E. Dye ${ }^{3}$, Brandy Dougherty-Hamod ${ }^{3}$, Matthew T. Moyer ${ }^{3}$

${ }^{1}$ Penn State Hershey Medical Center - Internal Medicine, Hershey, Pennsylvania, United States

2 Penn State Hershey Medical Center - Hematology and Oncology, Hershey, Pennsylvania, United States

${ }^{3}$ Penn State Hershey Medical Center - Gastroenterology and Hepatology, Hershey, Pennsylvania, United States submitted 11. March 2014 accepted after revision 23. June 2014

\section{Bibliography}

DOI http://dx.doi.org/

10.1055/s-0034-1377612

Published online: 19.9.2014

Endoscopy International Open 2014; 02: E256-E258

(c) Georg Thieme Verlag KG Stuttgart · New York

E-ISSN 2196-9736

\section{Corresponding author} Bradley W. Mathers Penn State Hershey Medical Center - Internal Medicine 500 University Dr

Hershey

Pennsylvania 17033

United States

Fax: +1-717-531-4977

bmathers@hmc.psu.edu
Endoscopic ultrasonography with fine needle infusion (EUS-FNI) of alcohol is the most reported method for EUS-guided tumor ablation. Several studies have reported successful EUS-guided ablation of pancreatic neuroendocrine tumors. However, these tumors have been relatively small $(<3 \mathrm{~cm})$. In this report, a 50-year-old man with a metastatic carcinoid tumor with a large porta hepatis mass was referred to our clinic for EUS-guided ethanol ablation. After two separate EUS-FNI ablations, there was a $36 \%$ reduction in tumor size $(9.0 \times 11.4 \mathrm{~cm}$ to $6.7 \times 9.8 \mathrm{~cm})$ with associated

\section{Introduction}

$\nabla$

Endoscopic ultrasonography with fine needle aspiration (EUS-FNA) is a key staging and diagnosis procedure for mediastinal and gastrointestinal lesions and malignancies. Increasingly, interventional EUS has been used for minimally invasive therapeutic procedures such as celiac plexus neurolysis, guide wire placement, brachytherapy seed placement for solid tumors, and chemoablation of lesions [1-3]. EUS with fine needle infusion (EUS-FNI) of alcohol is the most reported method for EUS-guided tumor ablation and works by a mechanism of cell membrane lysis, protein denaturation, and vascular occlusion leading to cell death [4]. While percutaneous ethanol ablation is well studied for the treatment of hepatic and adrenal tumors, EUS-guided ablation has proposed advantages for mediastinal, retroperitoneal and perigastric lesions due to proximity and real-time, Doppler enhanced, high resolution imaging of target lesions and surrounding structures, theoretically minimizing damage to nontumor tissue $[1,5]$. Previous studies have described successful EUS-guided ethanol ablation of pancreatic neuroendocrine tumors [1,5-7]. However, these tumors have been relatively small $(<3 \mathrm{~cm})$ and, to the best of our knowledge, there tumor lysis syndrome. Chromogranin A levels decreased from 460 to $132 \mathrm{ng} / \mathrm{mL}$. The patient reported complete resolution of abdominal pain within 2 weeks, but only mild improvement in flushing and diarrhea. In conclusion, large metastatic neuroendocrine tumors can be successfully treated with EUS-guided ethanol ablation. Evidence-based guidelines are needed with regard to the appropriate volume of ethanol injected in EUS-guided ablation to promote the efficacy and safety of this emerging procedure.

are no previous reported cases with larger tumors.

In this case, we describe a patient with symptomatic, metastatic carcinoid tumor with a large porta hepatis mass successfully treated with EUS-guided ethanol ablation.

\section{Case report}

$\nabla$

A 50-year-old man with a 21-year history of metastatic carcinoid tumor requiring a liver transplant 19 years previously, presented to our clinic with gradually worsening metastatic disease and a porta hepatis mass. Surveillance positron emission tomography (PET) images had shown an enlarging $9.0 \times 11.4 \mathrm{~cm}$ soft tissue mass in the region of the porta hepatis with much smaller metastatic deposits involving the lung, pancreas, and mediastinal lymph nodes ( $\bullet$ Fig. 1). Additionally, the patient was suffering from worsening right upper quadrant pain (thought to be due to mass effect) and carcinoid symptoms of flushing and diarrhea. Surgery and radiation were not believed to be viable treatment options and after a multidisciplinary evaluation, he was offered EUS-FNI with ethanol ablation with the intent to improve carcinoid symptoms and relieve mass effect on the biliary system and liver. 


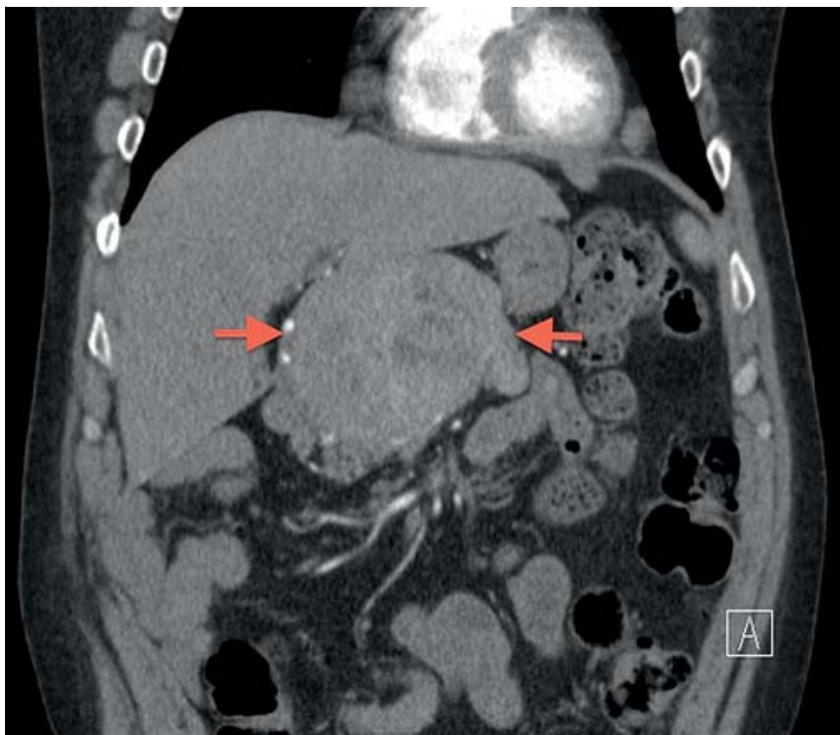

Fig. 1 Coronal abdominal computed tomography (CT) image performed before initial endoscopic ultrasonography with fine needle infusion (EUSFNI) revealed a large $9.0 \times 11.4 \mathrm{~cm}$ porta hepatis tumor (red arrows).

At the initial EUS-FNI, the large porta hepatis mass, which compressed both the extrahepatic biliary system and stomach, was evaluated via linear echoendoscope and a 22 gauge needle was introduced through a Doppler free window which was then used to perform three passes through the right, center, and left side of the tumor infusing $1 \mathrm{~mL}$ of $98 \%$ ethanol with each pass with $3 \mathrm{~mL}$ used in total ( Fig. 2). A repeat computed tomography (CT) scan 6 weeks later revealed minimal tumor reduction with a small central area of necrosis and the patient experienced no improvement in clinical symptoms.

The patient therefore underwent a second EUS-FNI with a much larger dose of $20 \mathrm{~mL}$ of $98 \%$ dehydrogenated alcohol infused into sequential sections of the tumor. This volume was determined after discussion with interventional radiology who recommended not exceeding $40 \mathrm{~mL}$ at one therapeutic session. The patient was admitted to the hospital observation unit, and within 12 hours, an aggressive tumor response ensued associated with massive tumor lysis syndrome (potassium 5.8, phosphorus 5.9, lactate dehydrogenase (LDH) 3192, uric acid 9.4, creatinine 3.2), intravascular hemolysis, and compromised liver function (International Normalized Ratio (INR) 3.7, total bilirubin 22.3, alanine transaminase (ALT) 3400, aspartate transaminase (AST) > 7500) requiring an 11-day hospitalization and aggressive fluid resuscitation.

The patient reported complete resolution of his baseline abdominal pain 2 weeks after discharge, was able to discontinue one of his hypertensive medications, but experienced only minimal improvement in his flushing and diarrhea. Chromogranin A levels fell from a pre-treatment level of 460 to $132 \mathrm{ng} / \mathrm{mL}$ and a followup CT scan revealed an interval decrease in tumor size from $9.0 \times$ $11.4 \mathrm{~cm}$ to $6.7 \times 9.8 \mathrm{~cm}$ ( $\bullet$ Fig. 3 ).

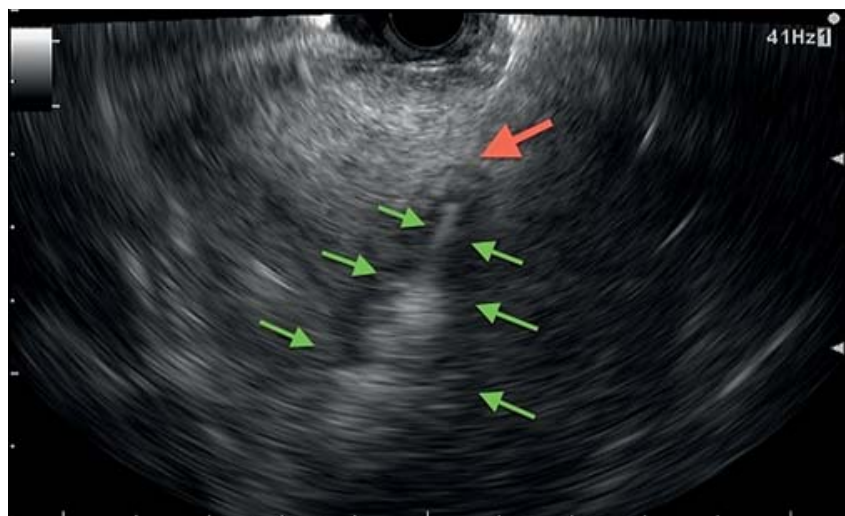

Fig.2 Still image of initial endoscopic ultrasonography with fine needle infusion (EUS-FNI) with needle tip (red arrow) being withdrawn through tumor while infusing $98 \%$ ethanol solution (green arrows).

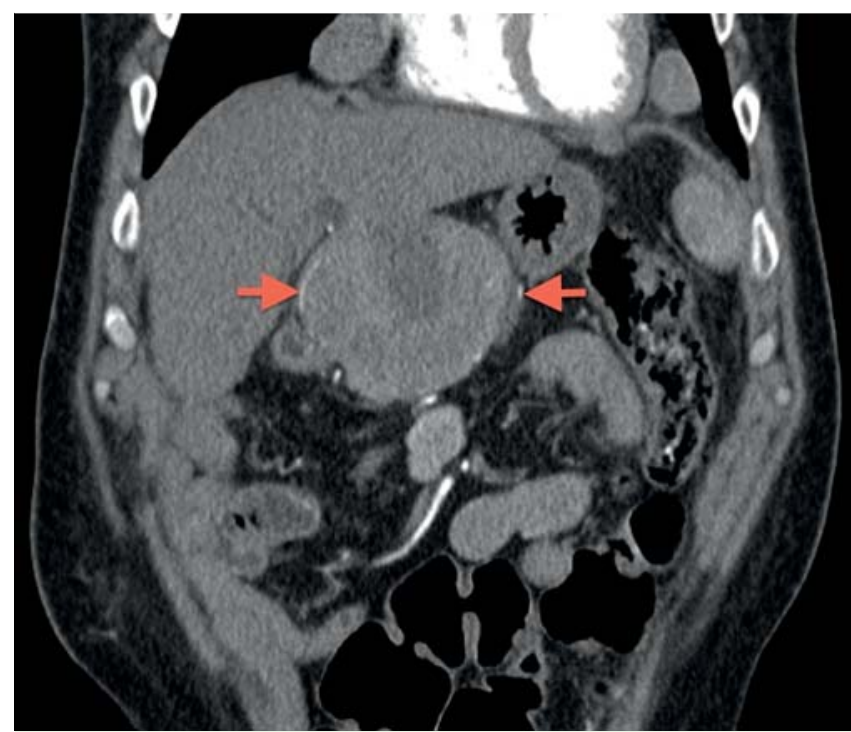

Fig. 3 Coronal abdominal computed tomography (CT) image performed 3 months after second endoscopic ultrasonography with fine needle infusion (EUS-FNI) revealed a $36 \%$ reduction in tumor size, now measuring $6.7 \times 9.8 \mathrm{~cm}$ (red arrows) with area of central necrosis.

\section{Discussion}

$\nabla$

In this case, we describe the relatively successful treatment of a large metastatic carcinoid with EUS-guided ablation. The patient achieved a $36 \%$ reduction in tumor size $\left(102.6 \mathrm{~cm}^{2}\right.$ vs $\left.65.7 \mathrm{~cm}^{2}\right)$ with complete resolution of tumor associated pain and minimal improvement in carcinoid type clinical symptoms. To the best of our knowledge, there are no previous reported cases of EUS ablation involving a tumor of this size. Levy et al. described eight cases of smaller insulinomas successfully treated with EUS-guided ablation (range $0.9-2.3 \mathrm{~cm}$ ) and Jürgensen et al. reported successful resolution of a $1.3 \mathrm{~cm}$ insulinoma via alcohol ablation [6, 8]. Muscatiello et al. also described treatment of a patient with multiple insulinomas $(1.1$ and $0.7 \mathrm{~cm}$ ) with ethanol ablation [5]. In these previous reports, the ablated tumors were smaller in size with a maximum size of $2.3 \mathrm{~cm}$ compared to the presented case of $11.4 \mathrm{~cm}$.

Previous studies have shown that multiple ethanol lavage treatments resulted in a greater decrease in size of tumors compared 
to only one lavage treatment [9]. This finding correlates with this case in which a significant improvement in both tumor size and clinical symptoms were not obtained until after a second lavage. We suspect that additional treatments would lead to further tumor reduction; however, as noted by Levy et al., a primary goal of relief of clinical symptoms rather than complete tumor resolution may be a more appropriate treatment goal to avoid overtreating and adverse events.

A consensus with regard to the appropriate volume of ablation agent to use in EUS-FNI does not exist. In the aforementioned studies, a wide range of alcohol volumes were used $(0.8-3.5 \mathrm{~mL})$ with no correlation between tumor size and ethanol volume injected. In our case, we first used a lower volume of $3 \mathrm{~mL}$ (or 0.03 $\mathrm{mL} / \mathrm{cm}^{2}$ ) followed by a significantly higher volume of $20 \mathrm{~mL}$ (or $0.20 \mathrm{~mL} / \mathrm{cm}^{2}$ ) and while the larger volumes did lead to improved tumor reduction and clinical response, it also led to significant tumor lysis syndrome. Guidelines for the determination of ablative agent to be infused per unit volume of tumor are needed to guide efficacy and safety in EUS-FNI procedures.

This case also illustrates an important but rare potential adverse event of EUS-guided ethanol ablation, particularly with large tumors. Earlier studies have reported mild to moderate adverse events such as mild pancreatitis, pseudocyst development, or perilesional necrosis $[5,6,8]$, however, there have been no previous reported cases of tumor lysis syndrome caused by EUSguided ethanol ablation. Tumor lysis syndrome, although more often associated with hematological malignancies, can be seen with treatment of large solid tumors and, when severe, can be life-threatening [10]. Therefore, we recommend assurance of proper intravenous access for prophylactic fluid hydration prior to, during, and after EUS-guided ablation of large neuroendocrine tumors to prevent such adverse event. Of note, a similar constellation of symptoms can be seen in Zieve's syndrome, in which ingestion of large amounts of ethanol can lead to intravascular hemolysis and liver failure. However, as we stayed significantly below the maximum dose of $40 \mathrm{~mL}$ ethanol, this was unli- kely to be Zieve's syndrome. We believe the patient's acute liver failure was secondary to ethanol ablation of surrounding hepatocytes near the tumor.

In conclusion, our case describes the successful treatment of a large metastatic carcinoid tumor with EUS-guided ethanol ablation. We advise appropriate caution when treating such large tumors with extended post-procedure monitoring for potential post-procedural adverse events such as tumor lysis syndrome. Evidence-based guidelines are needed with regard to the appropriate volume of ethanol injected in EUS-guided ablation to promote the efficacy and safety of this emerging procedure.

\section{Competing interests: None}

\section{References}

1 Zhang WY, Li ZS, Jin ZD. Endoscopic ultrasound-guided ethanol ablation therapy for tumors. World J Gastroenterol 14. 06 2013; 19: $3397-3403$

2 Wallace MB, Sabbagh LC. EUS 2008 Working group document: evaluation of EUS-guided ablation. Gastrointest Endosc 2009; 69: 59-63

3 Chennat J. Current status of endoscopic ultrasound guided ablation techniques. Gastroenterology 2011; 140: 1403-1409

4 Gelczer RK, Charboneau JW, Hussain S et al. Complications of percutaneous ethanol ablation. J Ultrasound Med 1998; 17: 531 -533

5 Muscatiello N, Salcuni A, Macarini L et al. Treatment of a pancreatic endocrine tumor by ethanol injection guided by endoscopic ultrasound. Endoscopy 2008; 40: E258 -E259

6 Jürgensen $C$, Schuppan $D$, Neser $F$ et al. EUS-guided alcohol ablation of an insulinoma. Gastrointest Endosc 2006; 63: 1059-1062

7 Deprez PH, Claessens A, Borbath I et al. Successful endoscopic ultrasound-guided ethanol ablation of a sporadic insulinoma. Acta Gastroenterol Belg 2008; 71: 333-337

8 Levy MJ, Thompson GB, Topazian MD et al. US-guided ethanol ablation of insulinomas: a new treatment option. Gastrointest Endosc 2012; 75: $200-206$

9 DiMaio CJ, DeWitt JM, Brugge WR. Ablation of pancreatic cystic lesions: the use of multiple endoscopic ultrasound-guided ethanol lavage sessions. Pancreas 2011; 40: 664-668

10 Howard S, Jones D, Pui C. The tumor lysis syndrome. N Engl J Med 2011; 364: $1844-1854$ 